\title{
Sulpiride and the potentiation of progestogen only contraception
}

\author{
M R PAYNE, P W HOWIE, A S MCNEILLY, W COOPER, M MARNIE, L KIDD
}

\begin{abstract}
A progestogen (norethisterone) and a dopamine antagonist (sulpiride) were given alone and in combination to volunteers to examine their effects on excretion of ovarian steroids. Compared with non-treatment cycles $(n=15)$, contraception with a progestogen alone $(n=10)$ was associated with increased excretion of oestrone and partial suppression of excretion of pregnanediol, suggesting partial luteinisation of unruptured follicles. By contrast, the combination of norethisterone and sulpiride $(n=9)$ suppressed both ovarian steroids to basal values, the suppression being even greater than with sulpiride alone $(n=5)$.
\end{abstract}

These results suggest that a combination of a progestogen with a dopamine antagonist might have a role in contraception.

\section{Introduction}

It has been suggested that contraceptives consisting solely of progestogen are seriously underused. ${ }^{1}$ They have advantages as contraceptives, the main one being that they are free of oestrogen. Their main drawbacks are that they are less reliable than combined oral contraceptives and are associated with irregular vaginal bleeding. If these problems could be overcome their use might increase considerably.

Progestogen only contraception appears to be more effective

\footnotetext{
Department of Obstetrics and Gynaecology, Ninewells Hospital and Medical School, Dundee DD1 9SY

M R PAYNE, MB, CHB, research fellow

$P$ W HOWIE, MD, professor

W COOPER, MSC, senior scientific officer

M MARNIE, MIST, chief technician

L KIDD, MB, CHB, senior registrar

Medical Research Council Reproductive Biology Unit, Edinburgh EH3 9EW A S MCNEILLY, DSC, senior scientist

Correspondence and requests for reprints to: Professor Howie.
}

during lactation (M R Payne, P W Howie, W C Cooper, M Marnie, unpublished findings), which may possibly be explained by the altered sensitivity of the hypothalamic-pituitary axis at this time. Women who are breast feeding are less sensitive to the positive feedback effects and more sensitive to the negative feedback effects of the ovarian steroids. ${ }^{2}$ Thus only a fairly small dose of ovarian steroid may be required to suppress secretion of gonadotrophin and stop ovarian cyclical activity during lactation.

In an attempt to improve acceptability and reliability we gave progestogen only contraception in combination with sulpiride. Sulpiride, a substituted benzamide, is a dopamine receptor blocker that causes an increase in secretion of prolactin and therefore reproduces the endocrine changes of lactation. ${ }^{3}$ Sulpiride itself has some contraceptive effect but is not sufficiently effective to be used reliably on its own. ${ }^{4}$ Our hypothesis was that when a progestogen (norethisterone) was given in combination with a dopamine antagonist the effect on the hypothalamic-pituitary axis would be similar to that seen in lactation, thereby making the combination more effective than either drug given alone.

\section{Volunteers and methods}

Five volunteer women of reproductive age were recruited for the study. All had normal menstrual cycles, and none had used a contraceptive pill or intrauterine contraceptive device within the previous three months. Each volunteer acted as her own control and was treated according to the following plan of study: cycle 1 , no treatment; cycles 2 and 3, norethisterone $350 \mu \mathrm{g}$ daily; cycle 4 , no treatment; cycles 5 and 6 , norethisterone plus sulpiride; cycle 7, no treatment; cycle 8 , sulpiride $50 \mathrm{mg}$ twice daily. Each volunteer collected an early morning urine specimen throughout the study and kept a menstrual calendar, noting any side effects possibly related to treatment. The urine samples were stored at $-20^{\circ} \mathrm{C}$ until assayed collectively.

On day 10 of cycles $1,2,5$, and 8 blood samples were taken for estimations of plasma prolactin concentrations. These were performed at the Medical Research Council Reproductive Biology Unit by radioimmunoassay as previously described ${ }^{5}$ and the results expressed as IU/l. The intra-assay variation was $7 \%$ and the interassay variation $9 \%$.

Urine oestrone-3-glucuronide and pregnanediol-3 $\alpha$-glucuronide (referred to hereafter as oestrone and pregnanediol) were measured by radioimmunoassay and the results expressed as $\mu \mathrm{g}$ and $\mathrm{mg} / \mathrm{g}$ creatinine respectively. ${ }^{6.8}$ The reagents were supplied by Dr P Samarajeewa, Courtauld Institute of Biochemistry, Middlesex Hospital Medical School. The intra-assay 
variations were $4 \%$ and $6 \%$, respectively, and the interassay variations $7 \%$ and $8 \%$.

The development of ovarian follicles and corpus luteums was monitored by ultrasound assessment using a Servo sector scanner. This was done regularly in two volunteers and periodically in the remaining three.

Comparisons between the cycles were made using paired and unpaired $t$ tests.

\section{Results}

The figure compares the mean (SE) excretions of oestrone and pregnanediol during the non-treatment cycles (cycles 1, 4, and 7) and the cycles of treatment with norethisterone alone (cycles 2 and 3), norethisterone plus sulpiride (cycles 5 and 6), and sulpiride alone (cycle 8). Results are grouped for each two day interval before menstruation for a total of 22 days.

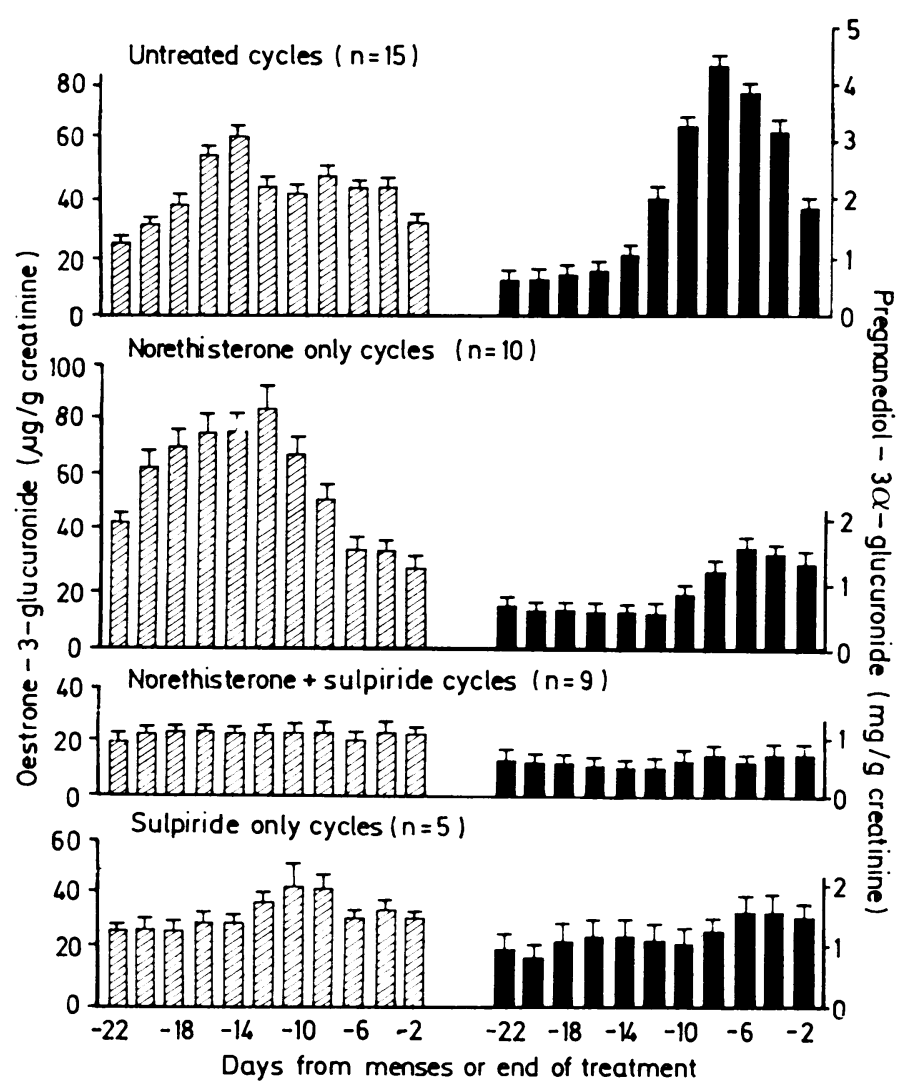

Mean (SE) excretions of oestrone-3-glucuronide (hatched bars) and pregnanediol-3 $\alpha$ glucuronide (closed bars) on paired days from menses or end of treatment.

When the secretion of ovarian steroids was completely suppressed and associated with amenorrhoea the results were calculated from the end of the period of treatment.

Non-treatment cycles-During the first non-treatment cycle (cycle 1) all volunteers showed biphasic excretion of oestrone with peaks in both follicular and luteal phases; a steep rise in excretion of pregnanediol occurred during the luteal phase in keeping with a normal pattern. ${ }^{9}$ In the nontreatment cycles 4 and 7 all volunteers showed immediate return to normal ovarian cycles. All 15 non-treatment cycles were combined and used as the normal reference range. The mean plasma prolactin concentration in the non-treatment cycles was 455 (SE 10) IU/l. In those non-treatment cycles in which the preovulatory follicle was measured by ultrasound the maximum diameter never exceeded $24 \mathrm{~mm}$.

Treatment with norethisterone alone-Compared with the non-treatment cycles, none of the cycles in which the women received norethisterone alone were normal. Seven of the 10 cycles showed considerably increased excretion of oestrone, which was associated with deficient excretion of pregnanediol in the premenstrual phase. Ultrasound measurements were made regularly in one of the cycles in which excretion of oestrone was increased, and the maximum diameter of the follicle reached $38 \mathrm{~mm}$. The other cycles showed relatively normal excretion of oestrone with grossly defective or basal production of pregnanediol, indicating failure of ovulation. The mean results reflected the pattern of the most common defect (the unruptured luteinised follicle), with significantly increased excretion of oestrone $(p<0.01)$ and defective excretion of pregnanediol $(p<0.01)$ compared with the untreated cycles. The mean plasma prolactin concentration was 442 (9) IU/1.

Treatment with norethisterone plus sulpiride-During treatment with norethisterone plus sulpiride oestrone and pregnanediol were suppressed to basal values, these being significantly lower $(p<0.01)$ than those found in the non-treatment cycles. Four of the nine periods of treatment were associated with amenorrhoea. No follicles were identified on ultrasound scanning. The mean plasma prolactin concentration was 2881 (95) IU/1.

Treatment with sulpiride alone-Compared with non-treatment cycles, treatment with sulpiride was associated with reduced excretion of both oestrone and pregnanediol, although the suppression was less complete than with the combination of norethisterone plus sulpiride. The concentrations of both hormones rose above basal values and in one cycle ovulation may have occurred, as indicated by a mid-cycle surge of luteinising hormone and a raised excretion of pregnanediol premenstrually. In some cycles there was evidence of some, but reduced, follicular development. The mean plasma prolactin concentration was 2459 (149) IU/1.

Side effects-One volunteer developed abdominal pain during treatment with norethisterone plus sulpiride and did not participate in the subsequent treatment cycles. The cause of the pain was not determined and settled within 36 hours without treatment. Ultrasound scanning at the time of pain showed no evidence of an ovarian cyst. Four volunteers reported slight drowsiness, and one reported loss of libido. Three volunteers had mild galactorrhoea, which was noticeable only on pressure. None of the symptoms were sufficient to warrant the treatment being stopped.

\section{Discussion}

The suppression of ovarian activity during breast feeding is nature's method of contraception. ${ }^{10}$ It would be very nice if a pharmacological method that reproduces the mechanisms of lactational infertility could be developed, and this underlying objective prompted this investigation.

Previous studies of the hormonal effects of contraceptives consisting solely of progestogen have shown that a variety of abnormal endocrine patterns may occur. In their study of norethisterone Landgren and Diczfalusy reported complete ovarian suppression in $16 \%$, increased follicular activity without luteal function in $23 \%$, and normal follicular activity with inadequate luteal phases in $21 \%$; the remaining $40 \%$ had oestradiol and progesterone profiles consistent with normal cycles. ${ }^{.1}$ Elstein $e t a l$, however, showed that even some of these cycles with apparently normal production of ovarian steroid may lack a surge of luteinising hormone. ${ }^{12} \mathrm{~A}$ recent report using ovarian ultrasound has shown a high incidence of functional ovarian cysts in users of the progestogen only pill, ${ }^{13}$ which is consistent with the predominant abnormality of high excretion of oestrone reported in our study. The alteration of endocrine function during treatment with the progestogen only pill may play an important part in its contraceptive effect. The degree of ovarian suppression, however, is unpredictable and incomplete, and this may explain the irregular vaginal bleeding and reduced contraceptive efficiency of the progestogen only pill. During lactation, however, the progestogen only pill is more reliable, presumably because of the changed sensitivity to oestrogen feedback induced by suckling. ${ }^{2}$ This increased contraceptive efficacy during lactation caused us to wonder whether sulpiride, a drug that mimics lactation by raising prolactin concentrations, might also augment the effects of the progestogen only pill.

Sulpiride alone has proved to be unsatisfactory as a contraceptive. Buvat et al studied 116 women over 1000 months of contraception with sulpiride and used excretion of pregnanediol, vaginal smears, and endometrial biopsy to assess ovarian function in some of the women, and basal body temperature in all of the women to indicate ovulation or luteal phase activity. ${ }^{4}$ Thirty eight of these women were taking high doses of sulpiride for psychiatric treatment, and its contraceptive effect was an addition to this. The doses of sulpiride used were 100 and $800 \mathrm{mg}$ daily. During an "observation phase," which lasted for from one to several months, the basal body temperature had to show no rise in the luteal phase for two cycles before the treatment was regarded as reliably achieving its contraceptive effect. Some women never achieved this. In the "safety 
phase" there were no method failures, but a progestogen was given with or without an oestrogen every two to three months to induce vaginal bleeding. Galactorrhoea was induced in all but four of the women, and drowsiness and sedation were common. In this study we found that the combination of a progestogen with a dopamine receptor blocker was more effective than either drug given alone. This may be because sulpiride, by sensitising the hypothalamicpituitary axis, rendered the action of norethisterone more effective. This combination may offer a new approach to contraception, and it may be possible to reduce the doses of both the dopamine antagonist and the progestogen considerably while still retaining a satisfactory contraceptive effect. It must be remembered that the progestogen only pill contains less progestogen than the combined pill, and a further reduction in progestogen content would be desirable after the doubts raised about its safety. ${ }^{14}$ Thus it may be possible to overcome the traditional problems of poor control of cycles and reduced effectiveness associated with progestogen only contraception. Reduction in dosage may reduce or eliminate the side effects associated with this combination, although the drugs were on the whole well tolerated.

Another potential use of this approach of combining the progestogen only pill with a dopamine antagonist would be its use during lactation. Badraoui and Hefnawi used sulpiride as a contraceptive with some apparent success during lactation. ${ }^{15}$ An improved supply of milk during lactation has been reported with administration of sulpiride, ${ }^{16}$ and a treatment regimen that acted as both a galactagogue and a contraceptive would be highly attractive. Because sulpiride is transferred in the breast milk its widespread use in lactating women cannot be recommended until more is known about its effects on the nursing infant. Further studies are required to determine the optimum dosage regimen and the possible side effects of this potentially promising new approach to contraception.
References

1 Graham S, Fraser IS. The progestogen-only mini-pill. Contraception 1982;26:373-88.

2 Baird DT, McNeilly AS, Sawers RS, Sharpe RM. Failure of estrogen induced discharge of luteinizing hormone in lactating women. Fourmal of Clinical Endocrinology and Metabolism 1979;49:500-90.

3 Delvoye $\mathrm{P}$, Taubert H-D, Jurgensen $\mathrm{O}$, et al. Evolution des gonadtrophines et de la progesterone sériques au cours d'une hyperprolactinemie induite par le sulpride (Dogmatil) pendant la phase sériques au cours d'une hyperprolactinemie induite par le sulpride (Dogmatil) pendant la phase
lutéale du cycle menstruel. Comptes Rendus Hebdomadaires des Séances de l'Academie des Sciences 1974;279, série D: 1463-6.

4 Buvat J, Decroix-Blacker C, Legal F, Gasnault JP. Mille mois de contraception par le sulpride. Revue Française de Gynécologie et d'Obstétrique 1976;71:53-61.

$5 \mathrm{McNeilly} \mathrm{AS,} \mathrm{Hagen} \mathrm{C.} \mathrm{The} \mathrm{prolactin,} \mathrm{LH,} \mathrm{FSH} \mathrm{and} \mathrm{TSH} \mathrm{response} \mathrm{to} \mathrm{a} \mathrm{combined} \mathrm{LHRH/TRH}$ test in normal male subjects and during the menstrual cycle. Clin Endocrinol 1974;3:426-35.

6 Baker ST, Jennison KM, Kellie. AE. The direct radioimmunoassay of oestrogen glucuronides in human female urine. Biochem $\mathcal{F}$ 1979;177:729-38.

7 Samarajeewa P, Cooley G, Kellie AE. The radioimmunoassay of pregnanediol-3 $\alpha$-glucuronide. $\mathcal{J}$ Steroid Biochem 1979;11:1165-71.

8 Wright K, Collins DC, Preedy JRK. Urinary excretion of oestrone glucosiduronate, 17 $\beta$ estradiol-17-glucosiduronate and estriol-16a-glucosiduronate. Significance of proportionate differences during the menstrual cycle. Steroids 1979;34:445-57.

9 Collins WP, Collins PO, Kilpatrick MJ, Manning PA, Pike JM, Tyler JPP. The concentrations of urinary oestrone-3-glucuronide, $\mathrm{LH}$ and pregnanediol-3a-glucuronide as indices of ovarian urinary oestrone-3-glucuronide, $\mathrm{LH}$ and

10 Howie PW, McNeilly AS. Effect of breast-feeding patterns on human birth intervals. 7 Reprod Fertil 1982;65:545-57.

11 Landgren B-M, Diczfalusy E. Hormonal effects of the $300 \mu \mathrm{g}$ norethisterone (NET) minipill. Contraception 1974;21:87-113.

12 Elstein M, Briston PG, Hewitt KJ, Kirk D, Miller $\mathrm{H}$. The effect of daily norethisterone $(0.35 \mathrm{mg})$ on cervical mucus and on urinary LH, pregnanediol and oestrogen levels. Br $\mathcal{O}$ Obstet Gynaecol $1976 ; 83: 165-8$

13 Toyob Y, Adams J, Jacobs HS, Guillebaud J. Ultrasonographic demonstration of increased frequency of functional ovarian cysts in progestogen-only pill users. Br $\mathcal{f}$ Obstet Gynaecol (in press).

4 Pike MC, Krailo MD, Henderson BE, Duke A, Roy S. Breast cancer in young women and the use of oral contraceptives: possible modifying effect of formulation and age at use. Lancet 1983;i:926-30.

15 Badraoui MHH, Hefnawi F. Ovarian function during lactation. In: Hafez ESE, ed. Human ovulation. Amsterdam: Elsevier/North Holland Biomedical Press, 1979.

16 Aono T, Shioji T, Aki T, Hirota K, Nomura A, Kurachi K. Augmentation of puerperal lactation by oral administration of sulpiride. Journal of Clinical Endocrinology and Metabolism 1979;48: 478-82.

(Accepted 31 May 1985)

\title{
Effect of isosorbide dinitrate, verapamil, and labetalol on portal pressure in cirrhosis
}

\author{
J G FREEMAN, J R BARTON, C O RECORD
}

\begin{abstract}
The effects on portal pressure of the vasodilatory drugs isosorbide dinitrate and verapamil and of an $\alpha$ and $\beta$ blocking agent, labetalol, were assessed in 21 patients with cirrhosis and portal hypertension. The wedged hepatic venous pressure gradient (wedged minus free hepatic venous pressures) was used as an index of portal pressure and was not significantly changed by treatment with labetalol $(n=5)$ but was significantly decreased by verapamil $(n=6 ; p<0.05)$ and isosorbide dinitrate $(\mathbf{n}=10 ; \mathbf{p}<0.01)$. Long term administration of isosorbide dinitrate also had a significant effect $(p<0.01)$.
\end{abstract}

\footnotetext{
Gastroenterology Unit, Royal Victoria Infirmary and University of Newcastle upon Tyne, Newcastle upon Tyne NE1 4LP

J G FREEMAN, MB, MRCP, senior registrar

J R BARTON, MB, MRCP, registrar

C O RECORD, DPHIL, FRCP, consultant physician

Correspondence to: Dr C O Record.
}

\section{Introduction}

Since the finding that propranolol lowers portal pressure ${ }^{1}$ there has been much interest in the pharmacological manipulation of pressure in the portal vasculature. The efficacy of propranolol in preventing variceal haemorrhage is controversial, ${ }^{2}$ and the bradycardia induced by propranolol may be potentially dangerous during acute episodes of bleeding. Nitroglycerin, a vasodilator, also lowers portal pressure when given both orally and intravenously but has a short half life. ${ }^{34}$ In this study we assessed the effect of the vasodilatory drugs isosorbide dinitrate and verapamil and an $\alpha$ and $\beta$ blocking agent (labetalol) on portal pressure in patients with cirrhosis with portal hypertension.

\section{Methods and results}

All patients had histologically confirmed cirrhosis and oesophageal varices. The wedged hepatic venous pressure gradient was used as an index of portal pressure and was measured by passing a catheter (Cordis No 8) into the right hepatic vein under fluoroscopic control. Wedged and free hepatic venous pressures were measured, the difference giving the wedged hepatic venous pressure gradient.

After suitable wedging of the catheter the patient was given one of the drugs under investigation. All drugs were given intravenously as a 